\title{
Block Length Determination of the Block Copolymer mPEG-b-PS Using MALDI-TOF MS/MS
}

This document is shared for only research purposes and cannot be distributed without the permission of the authors and the publisher. Please visit WWW.BECERGROUP.SEMS.QMUL.AC.UK/PUBLICATIONS.HTML to get more info on our research interests!!!

\author{
ANNA C. CRECELIUS, ${ }^{1,2}$ C. REMZI BECER, ${ }^{1,2,3}$ KATRIN KNOP, ${ }^{1,2}$ ULRICH S. SCHUBERT ${ }^{1,2,3}$ \\ ${ }^{1}$ Laboratory of Organic and Macromolecular Chemistry, Friedrich-Schiller-University Jena, \\ Humboldtstr. 10, Jena 07743, Germany \\ ${ }^{2}$ Dutch Polymer Institute (DPI), John F. Kennedylaan 2, 5612 AB Eindhoven, The Netherlands \\ ${ }^{3}$ Laboratory of Macromolecular Chemistry and Nanoscience, Eindhoven University of Technology, Den Dolech 2, 5600 MB \\ Eindhoven, The Netherlands
}

Received 19 May 2010; accepted 1 July 2010

DOI: $10.1002 /$ pola. 24223

Published online in Wiley Online Library (wileyonlinelibrary.com).

\begin{abstract}
The molar mass determination of block copolymers, in particular amphiphilic block copolymers, has been challenging with chromatographic techniques. Therefore, methoxy poly(ethylene glycol)-b-poly(styrene) (mPEG-b-PS) was synthesized by atom transfer radical polymerization (ATRP) and characterized in detail not only by conventional chromatographic techniques, such as size exclusion chromatography (SEC), but also by matrix-assisted laser/desorption ionization tandem mass spectrometry (MALDI-TOF MS/MS). As expected, different molar mass values were obtained in the SEC measurements depending on the calibration standards (either PEG or PS). In contrast, MALDI-TOF MS/MS analysis allowed the molar mass determination of each block, by the scission of the weakest point between the PEG and PS block. Thus, fragments of the individual blocks
\end{abstract}

could be obtained. The PEG block showed a depolymerization reaction, while for the PS block fragments were obtained in the monomeric, dimeric, and trimeric regions as a result of multiple chain scissions. The block length of PEG and PS could be calculated from the fragments recorded in the MALDI-TOF MS/MS spectrum. Furthermore, the assignment of the substructures of the individual blocks acquired by MALDI-TOF MS/MS was accomplished with the help of the fragments that were obtained from the corresponding homopolymers. (C) 2010 Wiley Periodicals, Inc. J Polym Sci Part A: Polym Chem 48: 4375-4384, 2010

KEYWORDS: block copolymer; MALDI-TOF MS/MS; mass spectrometry; poly(ethylene glycol); poly(styrene); structure; tandem MS
INTRODUCTION Amphiphilic block copolymers are of tremendous interest for various applications in which the combination of hydrophilic and hydrophobic properties are utilized, for example, as compatibilizers, dispersion stabilizers, and emulsifiers. Copolymers containing poly(ethylene glycol) (PEG) as hydrophilic and poly(styrene) (PS) as hydrophobic segment are used in many industrial applications, such as stabilizers, surfactants, and templates for the preparation of inorganic nanoparticles. ${ }^{1,2}$

One major breakthrough in block copolymer synthesis in the last two decades was the introduction of controlled radical polymerization techniques (CRP), such as atom transfer radical polymerization (ATRP). ${ }^{3}$ ATRP allows the synthesis of welldefined structures of copolymers in industry as well as on the laboratory scale. In ATRP, a transition metal compound acts as a carrier of a halogen atom in a reversible redox process. ${ }^{4}$

The detailed characterization of amphipilic copolymers is crucial, since the properties of such materials depend on the microstructures, such as the ratio of the corresponding block lengths, end group functionality, and the chain architecture. ${ }^{5}$ Traditional characterization techniques for this purpose are size exclusion chromatography (SEC) and proton-nuclear magnetic resonance $\left({ }^{1} \mathrm{H}\right.$ NMR) spectroscopy. However, since the introduction of soft ionization techniques, such as matrix-assisted laser desorption/ionization time-of-flight (MALDI-TOF) $^{6,7}$ and electrospray ionization (ESI), ${ }^{8}$ mass spectrometry (MS) has been placed in the focus of polymer analysis. ${ }^{9-12}$ The addition of tandem MS (MS/MS) analysis in combination with both soft ionization techniques has made the structure analysis of homopolymers, as well as copolymers easier. ${ }^{13-20}$ However, only a few reports exist about the MS/MS analysis of copolymers using MALDI-TOF ${ }^{21-23}$ and ESI. $^{24-30}$ Wesdemiotis et al. ${ }^{21}$ performed MALDI-TOF MS/MS measurements on the poly(fluoro oxetane)-co-(tetrahydrofuran) copolymer to obtain information regarding its architecture. The block copolymer mono- $n$-butyl ethers of poly(ethylene glycol)- $b$-poly(propylene oxide) (PEG- $b$-PPO) was analyzed by Weidner et $\mathrm{al}^{22}$ to determine the correct number of repeating units in the PEG and the PPO block of the 
TABLE 1 Summary of SEC and ${ }^{1} \mathrm{H}$ NMR Spectroscopy Data

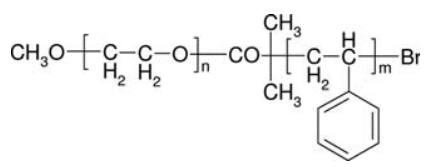

\begin{tabular}{|c|c|c|c|c|c|c|c|c|}
\hline $\begin{array}{l}\text { Sample } \\
\text { name }\end{array}$ & $\begin{array}{l}\text { Reac time } \\
\text { min }\end{array}$ & $\begin{array}{l}M_{\mathrm{n}, \mathrm{SEC}} \\
\mathrm{g} / \mathrm{mol} \\
\text { (PEG calibration) }\end{array}$ & PDI & $\begin{array}{l}M_{\mathrm{n}, \mathrm{SEC}} \\
\mathrm{g} / \mathrm{mol} \\
\text { (PS calibration) }\end{array}$ & PDI & NMR (n) & NMR (m) & $\mathrm{SEC}(\mathrm{m})$ \\
\hline Macroinitiator & - & 2,050 & 1.05 & 4,300 & 1.04 & 44 & 0 & 0 \\
\hline Sample 1 & 60 & 2,500 & 1.05 & 5,200 & 1.05 & 44 & 9 & 8 \\
\hline Sample 2 & 130 & 3,250 & 1.08 & 6,900 & 1.09 & 44 & 22 & 25 \\
\hline Sample 3 & 165 & 3,600 & 1.09 & 7,700 & 1.10 & 44 & 30 & 33 \\
\hline Sample 4 & 190 & 3,850 & 1.09 & 8,300 & 1.11 & 44 & 33 & 38 \\
\hline Sample 5 & 210 & 4,100 & 1.10 & 8,850 & 1.12 & 44 & 36 & 44 \\
\hline
\end{tabular}

copolymer. The end-groups and segment information about complex copolyester samples was obtained by Weidner et al. ${ }^{23}$ employing MALDI-TOF MS/MS analysis. Adamus ${ }^{24}$ investigated two random biodegradable copolyester macromolecules by applying ESI ion trap MS combined with $\mathrm{MS}^{n}$. Terrier et al. ${ }^{25}$ used ESI-MS/MS under low-energy conditions to investigate ammonium adducts of a series of linear triblock and glycerol derivative diblock copolyesters. First the authors performed tandem MS analysis of the homopolymers to identify the structure of the product ions and to propose a possible fragmentation pathway. The authors demonstrated with their work that it is possible to distinguish between copolyesters with the same composition in each repeating unit with inversed block sequences and that information about the block length can be readily obtained. Simonsick and Petkovska ${ }^{26}$ performed FT-MS ${ }^{n}$ for structural characterization and sequencing of a copolymer consisting of poly(glycidyl methacrylate) and methyl methacylate. Girod et al. ${ }^{27}$ determined accurately the PS block size in a PEG- $b$-PS block copolymer prepared by a nitroxide-mediated polymerization from the number of styrene losses as observed in the ESI-MS/MS spectrum. In an alternative fragmentation route the junction group between the two blocks could be structurally described. In contrast to the PS block, the PEG block did not show any specific dissociation. The authors expanded their studies by coupling liquid chromatography at critical conditions (LCCC) with on-line ESI tandem MS to characterize a PEG- $b$-PS block copolymer. ${ }^{28,29}$ Giordanengo et al. ${ }^{30}$ investigated by ESI-MS/MS as well as by NMR spectroscopy the microstructure of a poly(methacrylic acid)-poly(methyl methacrylate) copolymer. The random nature of the copolymer could only be detected by tandem MS analysis.

In this study, we first used the traditional methods, such as SEC, and ${ }^{1} \mathrm{H}$ NMR spectroscopy to characterize the block copolymer mPEG- $b$-PS, which was synthesized by ATRP; afterwards MALDI-TOF MS and MS/MS were applied to determine the size of both blocks. By using MALDI-TOF MS/MS analysis, the hydrolysis of both blocks, as described by Girod et al. ${ }^{31}$ was not necessary and therefore the analysis was much faster and easier.

\section{RESULTS AND DISCUSSION}

\section{Characterization of the Block Copolymer by SEC and ${ }^{1} \mathrm{H}$ NMR Spectroscopy}

The block copolymer mPEG- $b$-PS was synthesized utilizing the ATRP reaction. In total five copolymer samples were obtained besides the macroinitiator and were first characterized by SEC using either a PEG or a PS calibration and, secondly, by ${ }^{1} \mathrm{H}$ NMR spectroscopy. The results obtained from both characterization techniques are summarized in Table 1.

The difference in the calculated number average molar mass values $\left(M_{\mathrm{n}}\right)$ by using two different types of calibration standards are arising from the different hydrodynamic volumes of the copolymers obtained. As the calibration standards, either PEG or PS, do not have the same chemical structure to that of the copolymer samples, the relative $M_{\mathrm{n}}$ values should be in between the molar mass values calculated according to PEG or PS calibration. Small polydispersity index (PDI) values as expected from a controlled polymerization were obtained, as presented in Table 1. By integrating the signals of the ${ }^{1} \mathrm{H}$ NMR spectra, the $n$ and $m$ values for the two repeating units of $\mathrm{mPEG}-b$-PS were obtained. The integration of the peaks that belong to PEG in the ${ }^{1} \mathrm{H}$ NMR spectrum was taken as 44 repeating units and the corresponding PS content was calculated accordingly. Higher numbers of repeating units were observed at higher monomer conversions, as expected. The SEC graphs were also used to calculate the number of repeating units for PS in the block copolymers. The obtained $m$ values differ slightly from the values obtained by ${ }^{1} \mathrm{H}$ NMR spectroscopy. As depicted in Figure 1 , the hydrodynamic volume of the block copolymer is increasing at higher monomer conversions.

There is a shoulder appearing at higher elution time, which corresponds exactly to the PEG macroinitiator. The ratio of this shoulder to the formed polymer peak decreases due to the increased concentration of the polymer. This indicates that the shoulder belongs to unfunctionalized PEG macroinitiator. Nevertheless, the peaks in the SEC curves shift to the lower 


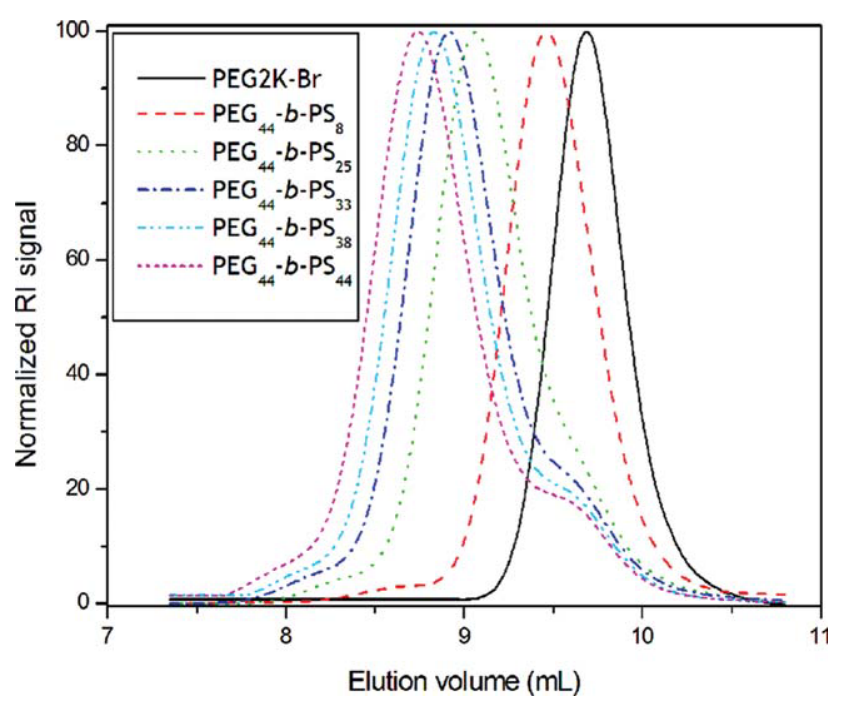

FIGURE 1 SEC curves from PEG-b-PS copolymer samples.

elution time proving the growth of the copolymer chain. It should be noted that there is another shoulder appearing at the lower elution time side. This peak corresponds to the chain-chain coupling reaction that is a side reaction during the radical polymerization of styrene. The impurities in the block copolymer, such as unfunctionalized PEG macroinitiator or the coupled block copolymer, have not been removed from the sample using dialysis or any other purification technique. In a follow-up study we are aiming to collect several fractions using 2D-SEC-LC to analyze each adduct, product or side product using MALDI-TOF MS and MS/MS techniques. This will allow us to detect the fingerprints of the polymerization.

\section{MALDI-TOF MS and MS/MS Analysis of Homopolymers}

The successful formation of the macroinitiator was investigated by MALDI-TOF MS analysis, as shown in Figure 2. The calculated $M_{\mathrm{n}}$ value for mPEG 2000 was $1907 \mathrm{~g} / \mathrm{mol}$ and for the macroinitiator $2122 \mathrm{~g} / \mathrm{mol}$; the corresponding PDI values

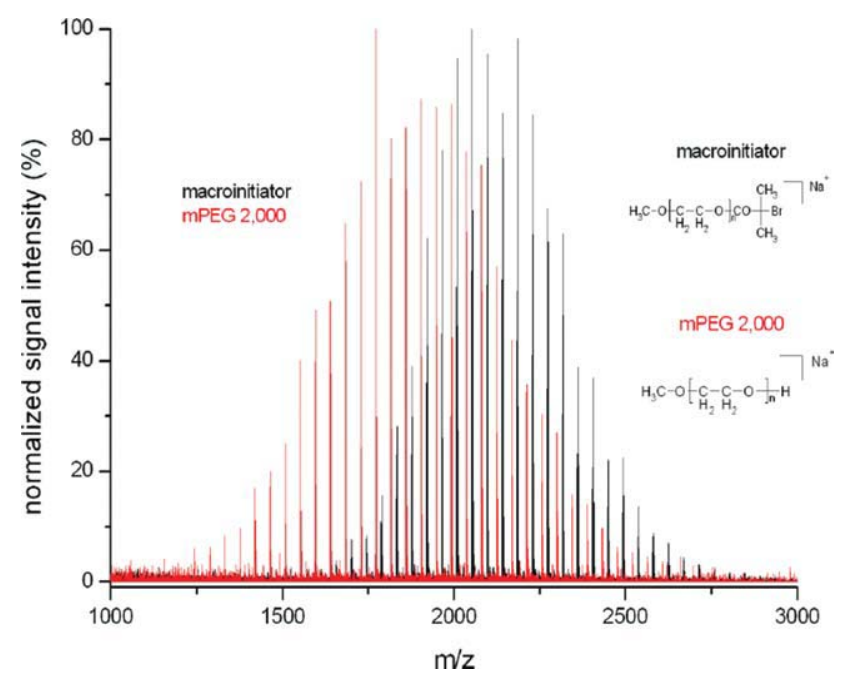

FIGURE 2 MALDI-TOF MS spectrum of mPEG 2000 and macroinitiator (matrix: DCTB, salt: Nal). using PolyTools for the determination for mPEG 2000 and the macroinitiator were 1.02 and 1.01 , respectively.

The experimental mass accuracy assigned for both polymers is around $m / z 0.1$, as presented in Table 2. This is within the mass accuracy achievable with the used instrument.

From the starting material mPEG 2000 and the macroinitiator MALDI-TOF MS/MS analysis was performed to evaluate which fragmentations can occur. The obtained fragments of the homopolymers could be later the basis for the elucidation of the structure of fragment ions in the copolymer analysis. To interpretate the fragment ions obtained for both homopolymers, the structures described by Hoteling et al. ${ }^{32}$ were used as possible starting points. Hoteling et al. ${ }^{32}$ proposed accordingly to Selby et al. $^{33}$ that a 1,4-hydrogen elimination and a 1,2-ethylene elimination are occurring as fragmentation routes. The dissociation of PEG was first described by Lattimer et al. using fast atom bombardment tandem MS. ${ }^{34}$ The ions according to these eliminations with the corresponding end groups could be determined in the recorded mass spectra for mPEG 2000 (Supporting Information Fig. 1 and Table 1) and for the macroinitiator (Supporting Information Fig. 2 and Table 2). The product ions arising from the dissociations of mPEG and the macroinitiator were labelled according to the nomenclature established by Lattimer et al. ${ }^{34}$ Starting with Supporting Information Figure 1, the MALDI-TOF MS/MS spectrum of MPEG 2000 using AgTFA as salt additives is presented herein. A parent ion with 41 repeating units was selected. The signals of the generated fragment ions in Supporting Information Figure 1(a) reveal a depolymerization reaction. By zooming into the MALDI-TOF MS/MS spectra, as presented in Supporting Information Figure 1(b), it could not be determined accurately if the fragment ions $\mathrm{C}$ and $\mathrm{A}^{\prime}$ are formed or if they can be explained by the silver isotopes of the fragment series $B$ and $\mathrm{C}^{\prime}$, respectively, since the detected ions are only $2 \mathrm{~m} / \mathrm{z}$ values apart from each other. The MALDI-TOF MS/MS analysis was also performed with $\mathrm{NaI}$, and more or less the same fragments were obtained, except fragment A, as in the AgTFA cationized sample. More detailed information can be found in Supporting Information Figure 1.

mPEG 2000 has a methoxy group on one end and a hydroxyl group on the other end. The hydroxyl group is reacted by $\alpha$ bromo-iso-butyryl bromide to yield the PEG macroinitiator. As mentioned earlier, the macroinitiator was analyzed as well by MALDI-TOF MS/MS using DCTB as matrix with the addition of NaI. A parent ion with 41 repeating units corresponding to a $\mathrm{m} / \mathrm{z}$ value of 2008 was selected and the

TABLE 2 Measurements of the Mass Accuracy Obtained for the MALDI-TOF MS Analysis of MPEG 2000 and the Macroinitiator (Fig. 2)

\begin{tabular}{llll}
\hline Species & $m / z^{\text {theo }}$ & $m / z^{\text {exp }}$ & Error $(m / z)$ \\
mPEG 2000 & 1772.04 & 1771.92 & 0.12 \\
Macroinitiator & 1919.99 & 1919.86 & 0.13
\end{tabular}




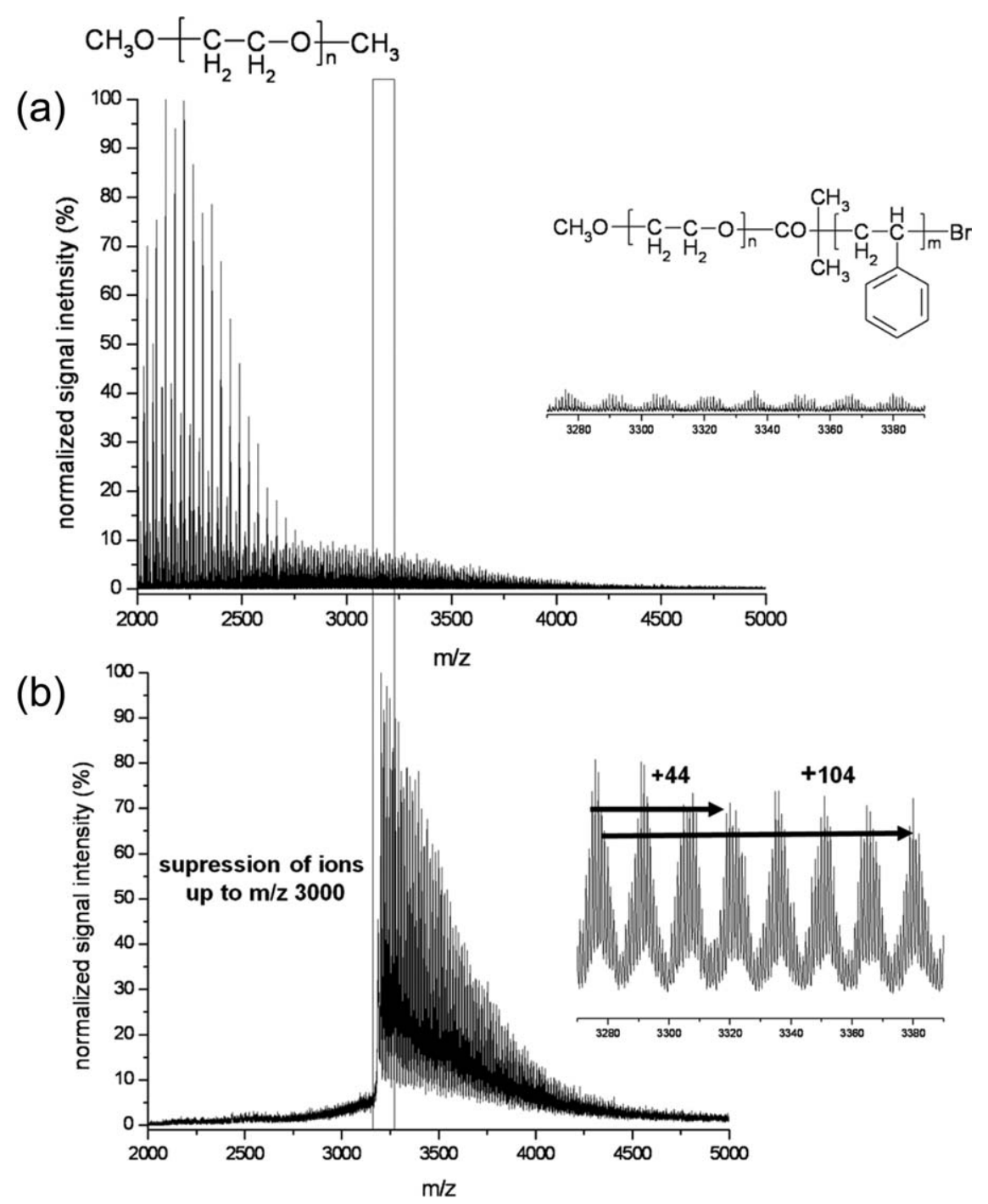

FIGURE 3 MALDI-TOF MS spectra of mPEG-b-PS (copolymer 1) in (a) normal mode, and (b) suppressing of ions up to $m / z$ 3000. DCTB was used as matrix and AgTFA as salt additive. resulting MALDI-TOF MS/MS spectrum can be found in Supporting Information Figure 2. The zoom displayed in Supporting Information Figure 2(b) in connection with Supporting Information Table 2 shows the formation of seven fragment series $\left(B, B^{\prime}, B^{\prime \prime}, C, C^{\prime}, C^{\prime \prime}\right.$ and $\left.C^{\prime \prime \prime}\right)$. The fragment series $B, B^{\prime}, C$, and $C^{\prime}$ are also detected when mPEG 2000 is fragmented, as presented in Supporting Information Figure 1(b), only as sodium adducts instead of silver adducts. Two additional fragment series $\mathrm{B}^{\prime \prime}$ and $\mathrm{C}^{\prime \prime}$ could be obtained, showing the characteristic end group of the macroinitiator. At first instance a bromoester moiety was expected as end group, but the distinct isotopic pattern of the bromine atom was not found and cleavage of the bromine atom from the parent ion of the macroinitiator was observed, since a fragment ion with $m / z 1929$ is presented in Supporting Information Figure 2. Fragment series $\mathrm{C}^{\prime \prime \prime}$ is as well formed, which contains the unsaturated end group $\mathrm{CH}_{3}-\mathrm{C}=\mathrm{CH}_{2}$. Additionally, a fragment ion, in which the whole bromoester group was cleaved off, was also recorded at the $m / z$ value of 1859 . Fragment series having a hydrogen atom as end group could be identified. This explains how fragment series $\mathrm{B}^{\prime}$ and $\mathrm{C}$ could be formed bearing a hydrogen atom as end group.

After the MALDI-TOF MS/MS analysis of MPEG 2000 and the macroinitiator was discussed in detail, now the tandem MS analysis of PS will be in the focus of this contribution. PS with a 1,1-dimethylacetic acid ethylester group as initiator moiety and a bromine atom as end group was selected. The matrices DCTB and dithranol and the salt additives NaI and AgTFA were tested for the MALDI-TOF MS analysis of PS. The highest signal-to-noise levels showed the matrix dithranol in combination with AgTFA for the MALDI-TOF MS measurement of PS (spectrum not shown). The corresponding MALDI-TOF MS/MS spectra of PS are presented in Supporting Information Figure 3. As parent ion the silver adduct with 35 repeating units of PS was selected with a $m / z$ value of 3864. In the parent ion the bromine as end group was cleaved off and a radical was formed. The instability of the $\mathrm{C}-\mathrm{Br}$ bond is extensively discussed by Borman et $\mathrm{al}^{35}$ on PMMA synthesized by ATRP. The authors showed that methyl bromide gets lost and a ring-closed lactone end group is 

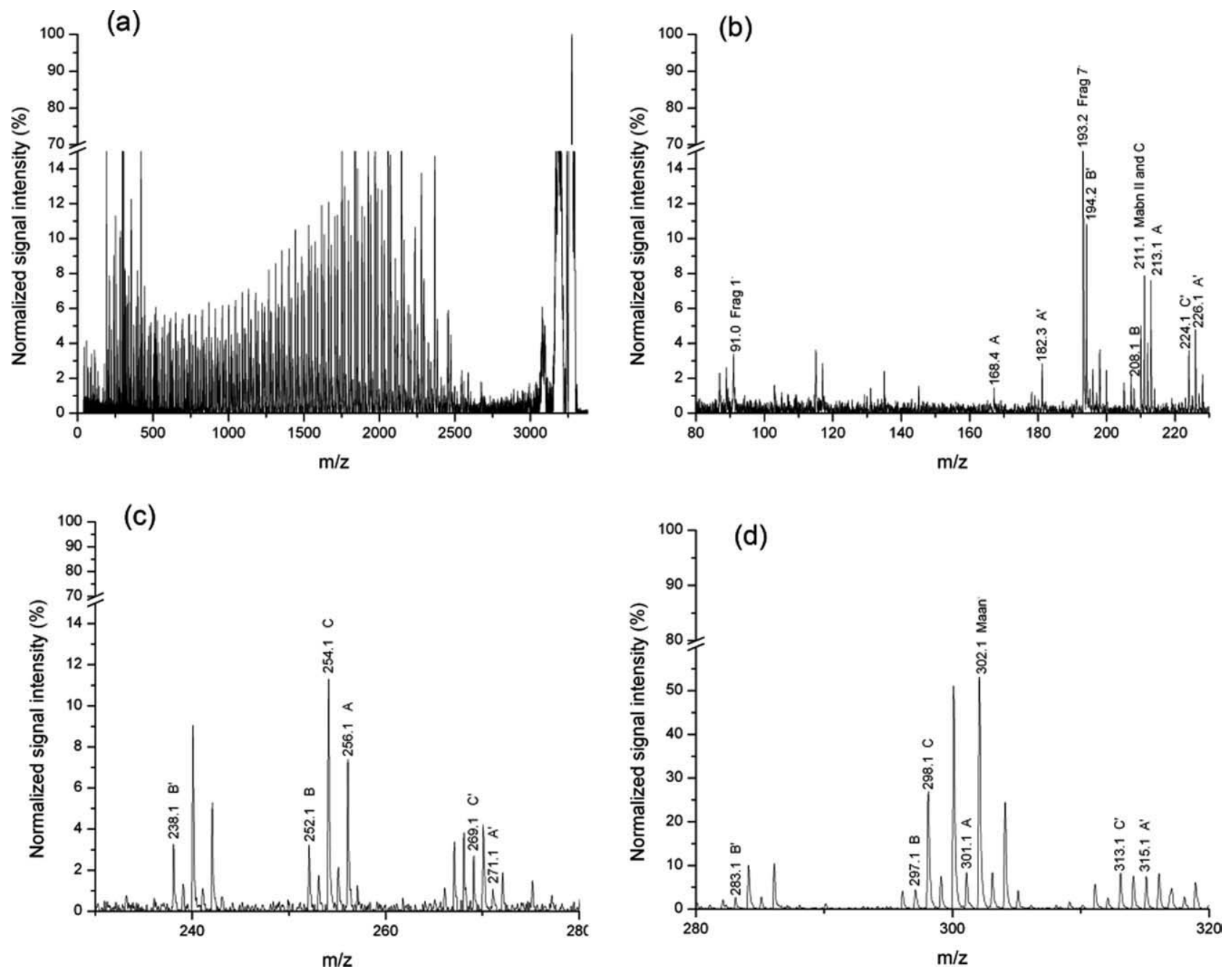

FIGURE 4 MALDI-TOF MS/MS spectra of mPEG- $b$-PS using the highest isotopic peak of $\mathrm{mPEG}_{59}-\mathrm{PS}_{4}$ with $\mathrm{m} / z 3305$ as $\mathrm{precursor}$ ion; (a) complete, (b) monomeric, (c) dimeric, and (d) trimeric region of the mass spectrum.

formed during the MALDI process. The generated fragment ions are annotated in the zoom shown in Supporting Information Figure 3(b) and the structures are summarized in Supporting Information Table 3. The nomenclature, as introduced by Gies et al., ${ }^{36}$ was selected for labeling the fragments produced by the MALDI-TOF MS/MS analysis.

In the elucidation of the structures of the recorded peaks, the reports of Gies et al. ${ }^{36}$ as well as Polce et al. ${ }^{37,38}$ were used as assistance. All three studies came to the conclusion that free radicals are formed and undergo further fragmentations. In Supporting Information Table 3 only the fragment ions labeled with Ian. and Ibn II show the characteristic 1,1dimethylacetic acid ethylester group as initiator group, all other fragment ions underwent scission of this initiator moiety and could have also been generated if a butyl group would have been used as initiator instead, corresponding to the measurements by Gies et al. ${ }^{36}$ and Polce et al. ${ }^{37} \mathrm{~A}$ detailed mechanism about the formation of these specific product ions has been reported by Gies et al. ${ }^{36}$
The studied block copolymer exhibits the same Br termination, although the cleavage of the $\mathrm{C}-\mathrm{Br}$ bond does not occur, since the selected precursor ion of the MALDI-TOF MS/MS analysis with a $m / z$ value of 3305 corresponds to the intact silver-containing molecule with 59 PEG and 4 PS repeating units, as shown later in this contribution. One possible explanation for this unexpected phenomenon can be that the $\mathrm{C}-\mathrm{Br}$ bond cleavage is time dependent. PS was synthesized 6 months before the block copolymer was produced and both compounds were analyzed by MALDI-TOF MS and tandem MS at the same date.

\section{Characterization of the Block Copolymer by MALDI-TOF MS and MS/MS Analysis}

The MALDI-TOF MS spectrum of the copolymer sample 1 is presented in Figure 3. From Figure 3(a), it is obvious that the unfunctionalized PEG, which is the remaining reagent from the synthesis of the macroinitiator, is masking the signals of the copolymer sample. This effect can be overcome 
TABLE 3 Structural Assignments for the Peaks in the MALDI-TOF MS/MS Spectra of Figure 4, Fragments of the PS Block, and mPEG Block

\begin{tabular}{lll}
\hline Label & lons Observed $(\mathrm{m} / \mathrm{z})$ \\
Frag 1 & 91.0 & Schematic Representation of the Structures \\
Frag 7 & 193.2
\end{tabular}

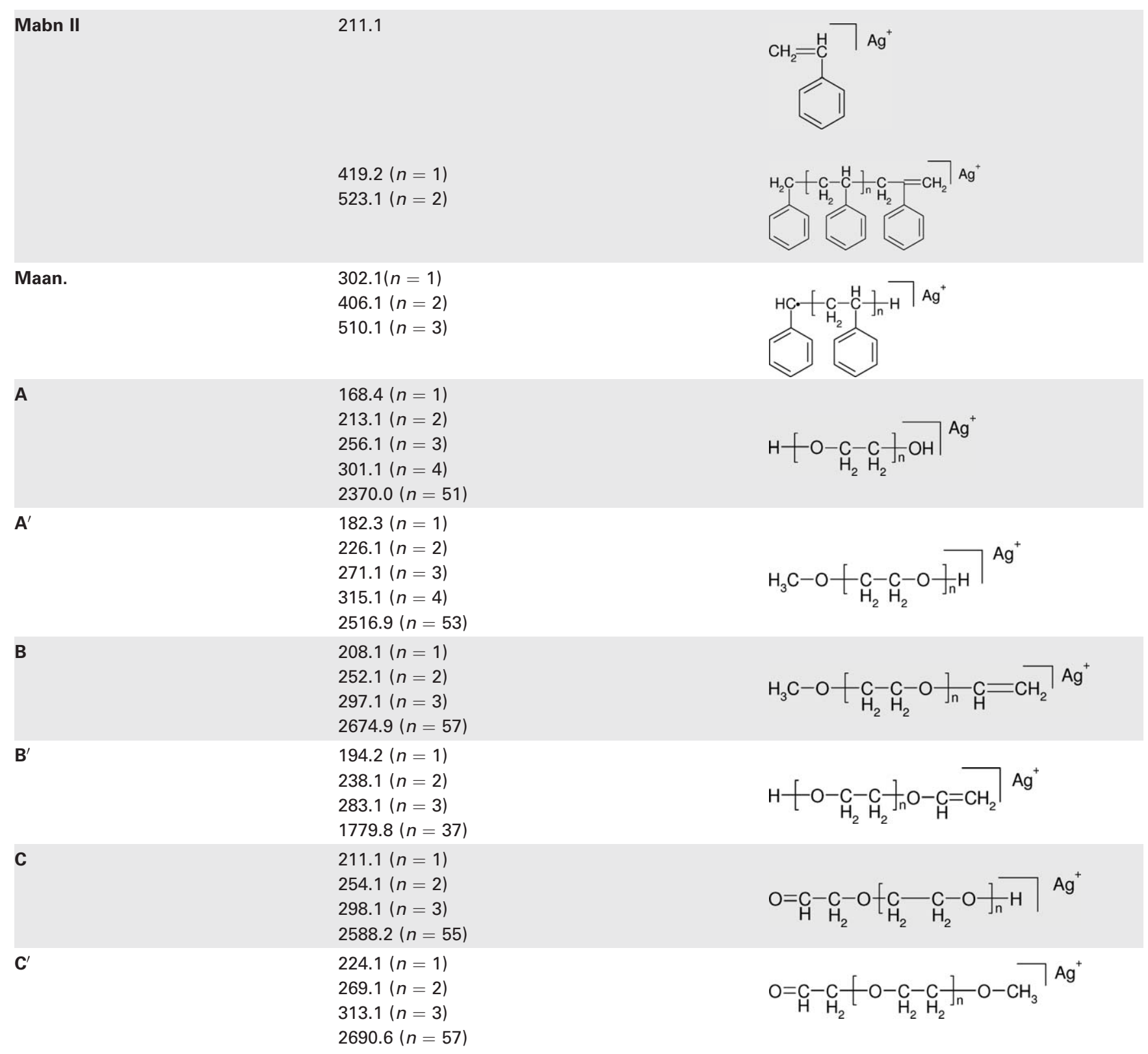


TABLE 4 Measurements of the Mass Accuracy of the Fragment Peaks Presented in the MALDI-TOF MS/MS Spectra Shown in Figure 4

\begin{tabular}{lrrl}
\hline Species & $m / z^{\text {theo }}$ & $m / z^{\text {exp }}$ & Error $(m / z)$ \\
Frag 1. & 91.0 & 91.1 & 0.1 \\
Frag 7. & 193.1 & 193.2 & 0.1 \\
Mabn II & 211.0 & 211.1 & 0.1 \\
Maan. & 302.0 & 302.1 & 0.1 \\
A & 301.0 & 301.1 & 0.1 \\
A $^{\prime}$ & 315.0 & 315.1 & 0.1 \\
B & 297.0 & 297.1 & 0.1 \\
B $^{\prime}$ & 283.0 & 283.1 & 0.1 \\
C $^{\prime}$ & 211.0 & 211.1 & 0.1 \\
C $^{\prime}$ & 313.0 & 313.1 & 0.1
\end{tabular}

by suppressing the ions up to $m / z 3000$ reaching the detector.

By using this approach, intense signals of the copolymer sample could be recorded, as shown in Figure 3(b). The zoom of the MALDI-TOF MS spectrum reveals the assignment of the repeating units of both blocks (PEG with $\mathrm{m} / \mathrm{z} 44$ and PS with $m / z$ 104). The remaining copolymer samples have been analyzed in the same manner by MALDI-TOF MS. The corresponding MALDI-TOF MS spectra can be found in Supporting Information Figure 4. With rising sample number, the copolymer distribution is shifting to higher $m / z$ values and the MALDI-TOF MS analysis is more and more difficult. Hence, a pre-fractionation, such as two-dimensional liquid chromatography, before the MALDI-TOF MS measurements, would be highly favorable.

The MALDI-TOF MS/MS analysis was performed on the highest isotopic peak $(\mathrm{m} / z$ 3305) of $\mathrm{mPEG}-b$-PS with 59 repeat units of $m$ PEG and 4 repeat units of PS. Since the window of the precursor ion selector could only be closed up to $m / z 4$, without losing the precursor ion signal, side products, such as $\mathrm{mPEG}_{52}-b-\mathrm{PS}_{7}$ with $\mathrm{m} / z 3309$ (highest isotopic peak) and $\mathrm{mPEG}_{66}-b-\mathrm{PS}_{1}$ with $\mathrm{m} / z 3301$ (highest isotopic peak) could also be fragmented in the analysis. If this would be the case, the fragmentation of $\mathrm{mPEG}_{66}-b-\mathrm{PS}_{1}$ should reveal a different pattern of fragments due to the short PS block. However, patterns correlated to such a fragmentation could not be found. The only possibility to overcome this phenomenon is the use of an instrument with higher resolution, such as a fourier transform ion cyclotron resonance (FTICR) mass spectrometer or an orbitrap. The resulting mass spectra from the instrument used in this study are presented in Figure 4.

Corresponding structures could be assigned to nearly all peaks in the monomeric, dimeric, and trimeric region of the mass spectrum [see Fig. 4(b-d)]. The structures, which are summarized in Table 3, could be found with the help of the MALDI-TOF MS/MS spectra of the homopolymers. The assignment of the fragment ions was performed over the whole available $m / z$ range, but are not included in Table 3 .

To show the validity of our assignments, first the mass accuracy for the identified fragments was calculated. The results are presented in Table 4 . The obtained mass accuracy was $m / z 0.1$, which lies in the specifications of the used mass detector. Additionally, the measured isotopic patterns were compared with the calculated ones for the identified fragments and are shown in Supporting Information Figure 5. In some cases, overlapping $\mathrm{m} / \mathrm{z}$ value peaks are obtained, making the identification of the fragments demanding. However, in most cases a series of fragments with differing numbers of repeating units was observed and used to judge the assignment.

Obviously only fragments of the mPEG block (Table 3) and the PS block (Table 3) could be detected in the MALDI-TOF MS/MS spectrum of MPEG- $b$-PS, which means that there were no signals detected correlated to fragments bearing the mPEG block as well as the PS block. From this phenomenon it can be concluded that a scission between both blocks in the tandem MS mode occurs.

Three scissions, as shown in Scheme 1, are possible. The first option shown in Scheme 1 (a) is that a 1,4-hydrogen elimination starts from the end of the mPEG block, so that the fragment series $\mathrm{B}$ and $\mathrm{X}$ are formed. The fragment series $\mathrm{B}$, as presented in Table 3, could be identified in the corresponding MALDI-TOF MS/MS spectrum of mPEG- $b$-PS. Fragment series $\mathrm{X}$ is only detectable with one repeating unit at the $m / z$ value of 420.1 . It might be that it fragments further in the tandem MS mode and is not observed in the corresponding MALDI-TOF MS/MS spectrum, since it possibly does not ionize with $\mathrm{Ag}^{+}$. A second 1,4-hydrogen-elimination can occur, as presented in Scheme $1(\mathrm{~b})$, so that fragment series $\mathrm{X}$ and $\mathrm{C}^{\prime}$ are formed as a consequence. Fragment series $\mathrm{X}$ could only be identified with one repeating unit in the corresponding MALDI-TOF MS/MS spectrum, since it possibly fragments further and does not ionize with $\mathrm{Ag}^{+}$. Fragment series $C^{\prime}$ could be easily detected in the MALDI-TOF MS/MS spectrum (see Fig. 4). The third option, as presented in Scheme $1(\mathrm{c})$, is the McLafferty rearrangement, where a hydrogen atom is separated from the block copolymer. Here again, as shown in Scheme 1(a), the fragment series B is formed as well as fragment series $\mathrm{X}$, which could not be found in the acquired MALDI-TOF MS/MS spectrum. As in the recorded MALDI-TOF MS/MS spectrum (Fig. 4 and Table 3) fragment series of MPEG with 57 repeating units could be observed, the fragmentation route as presented in Scheme $1(\mathrm{a}, \mathrm{b})$ is possibly favored. If a McLafferty rearrangement would take place one could expect to see 58 repeating units of fragment series $\mathrm{B}$ and at least fragment $\mathrm{X}$ with one repeating unit.

As mentioned earlier, no fragment series containing both repeating units of $\mathrm{MPEG}$ and PS could be observed. This was proven by acquirering MALDI-TOF MS/MS spectra from precursor ions containing the same number of mPEG repeating units and an increasing number of PS repeating units, as 
(a)<smiles>[X]C(C)(C=C)OCCC(C)(C)OC</smiles>

(b)
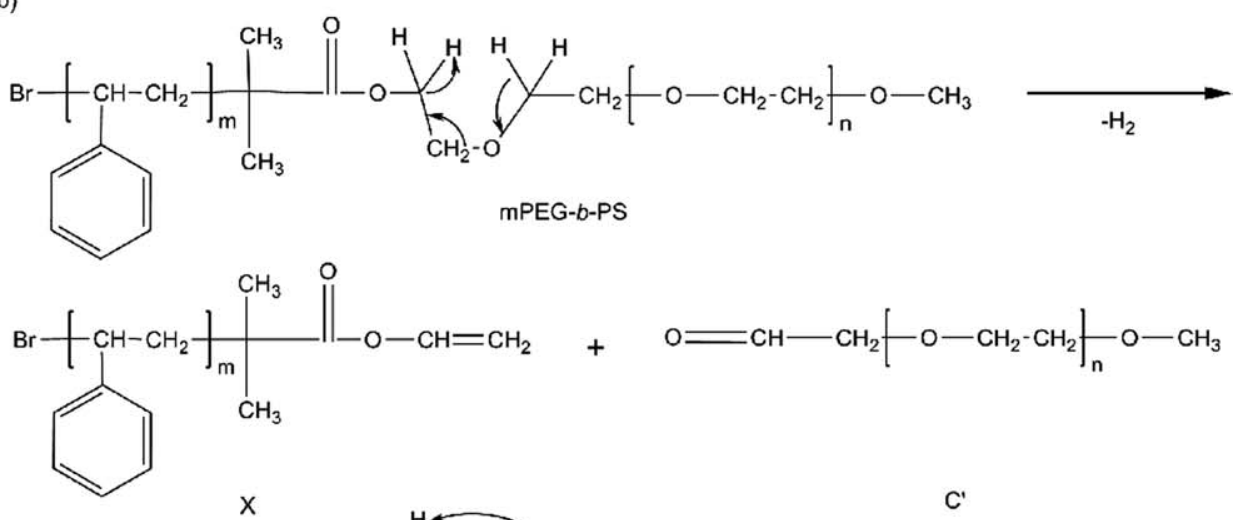

(c)

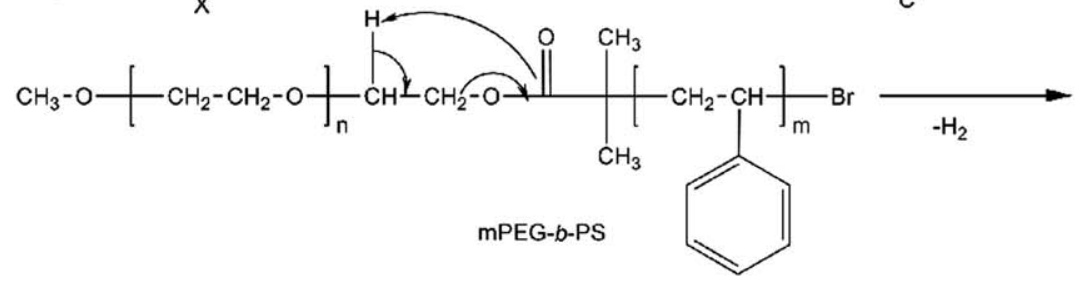

$\mathrm{CH}_{3}-\mathrm{O}-\left[-\mathrm{CH}_{2}-\mathrm{CH}_{2}-\mathrm{O}-\right]_{\mathrm{n}} \mathrm{CH}=\mathrm{CH}_{2}+$<smiles>CC(C)(CC(C)(C)C(C)(C)Br)C(=O)O</smiles>

$\mathrm{x}$
SCHEME 1 Proposed scission of the mPEG block from the PS block. (a), (b) 1,4-Hydrogen-elemination from each side of the copolymer chain, and (c) McLafferty rearrangement. presented in Figure 5. With increasing the repeating units of PS, the gap in the mass spectrum, where no fragments were obtained at higher molar masses, increased. Therefore a scission between both blocks is proposed as a result for the tandem MS analysis of mPEG- $b$-PS.

\section{EXPERIMENTAL}

\section{Materials}

Styrene $(\geq 99 \%$, Aldrich) and anisole (Fluka) were passed through neutral alumina oxide column before use. $\mathrm{CuBr}$ (99.999\%, Aldrich), $\alpha$-bromo isobutyryl bromine (98\%, Aldrich), ethyl-2-bromoisobutyrate (98\%, Aldrich), poly(ethylene glycol) methyl ether $\left(M_{\mathrm{n}}=2000 \mathrm{~g} / \mathrm{mol}\right.$, flakes $)$,
$N, N, N^{\prime}, N^{\prime \prime}, N^{\prime \prime}$-pentamethyldiethylenetriamine (99\%, Aldrich), and triethylamine ( $\geq 99 \%$, Aldrich) was used as received.

\section{Instruments}

For the determination of the monomer conversions, gas chromatography (GC) measurements were performed on a Shimadzu GC used with a Trace column RTX-5 and an autosampler. ${ }^{1} \mathrm{H}$ NMR spectroscopy was recorded on a Bruker Avance $250 \mathrm{MHz}$ in deuterated methylene chloride. The chemical shifts were calibrated with respect to tetramethylsilane (TMS). SEC was measured on an Agilent system which is equipped with triple detectors that are a diode array detector, a reflactive index detector, and a multiangle light scattering detector. Two PSS SDV ( $5 \mu$ pore size) columns were placed in series. DMA with $5 \mathrm{mmol} \mathrm{LiCl}$ was used as eluent 


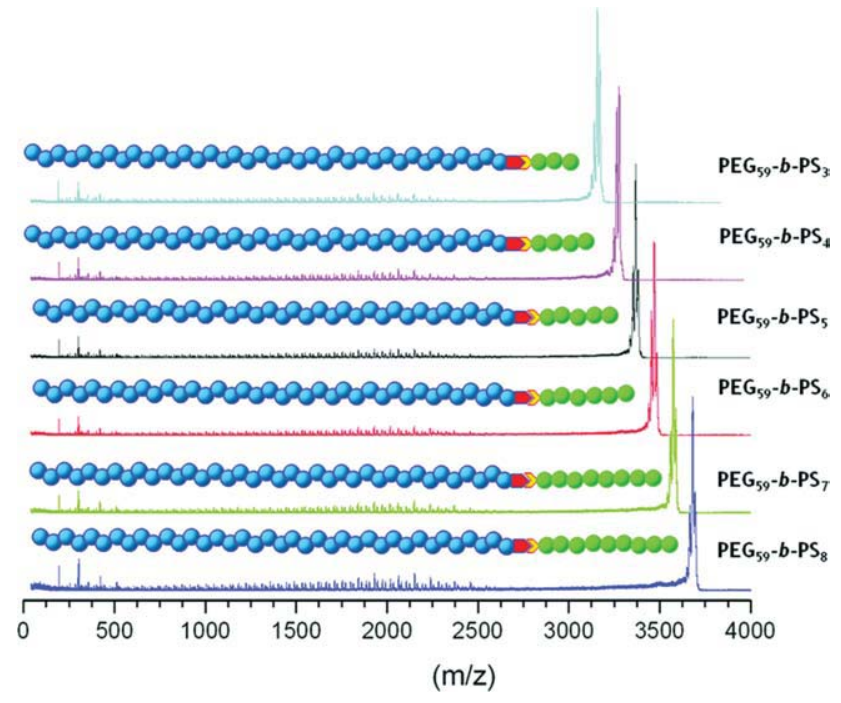

FIGURE 5 MALDI-TOF MS/MS spectra of precursor ions containing 59 repeating units of $\mathrm{MPEG}$ and an increasing number of PS repeating units. The selected precursor ions are schematically represented between the spectra. DCTB was used as matrix and AgTFA as salt additive. [Color figure can be viewed in the online issue, which is available at wileyonlinelibrary. com.]

at $1 \mathrm{~mL} / \mathrm{min}$ flow rate and the column oven was set to 50 ${ }^{\circ} \mathrm{C}$. An Ultraflex III TOF/TOF (Bruker Daltonics, Bremen, Germany) was used for the MALDI-TOF MS and MS/MS analysis. The instrument is equipped with a Nd:YAG laser and a collision cell. For the MS/MS mode, argon was used as collision gas at a pressure of $2 \times 10^{-6}$ mbar. The instrument was calibrated before every measurement with an external standard PMMA from PSS Polymer Standards Services GmbH (Mainz, Germany). MS data were processed using PolyTools 1.0 (Bruker Daltonics).

\section{CONCLUSIONS}

The block length of the copolymer mPEG- $b$-PS could be determined in an accurate and reliable manner by performing MALDI-TOF MS/MS analysis, since a dissociation of the PEG block was leading to the separation of both blocks. The PEG block showed a depolymerization reaction, while for the PS block fragments in the monomeric, dimeric, and trimeric regions were observed. This fast and easy to perform measurement does not require the hydrolysis of mPEG- $b$-PS followed by the independent MALDI-TOF MS analysis of each individual block to determine the block length of the copolymer. Future experiments will be concentrated on the purification of the block copolymers by performing two-dimensional liquid-chromatography before MALDI-TOF MS and tandem MS analysis.

The authors thank the Dutch Polymer Institute (DPI, technology area high-throughput-experimentation) and the Thüringer Ministerium für Bildung, Wissenschaft und Kultur (grant no.
B515-07008) for the financial support of this study. They also thank Bruker Daltonik GmbH for their cooperation.

\section{REFERENCES AND NOTES}

1 Dixit, S. G.; Mahadeshwar, A. R.; Haram, S. K. Colloids Surf A $1998,133,69-75$.

2 Westman, A.; Huthfehre, T.; Demirev, P.; Sundqvist, B. U. R. J Mass Spectrom 1995, 30, 206-211.

3 Kamigatio, M.; Ando, T.; Sawamoto, M. Chem Rev 2001, 101, 3689-3745.

4 Matyjaszewski, K.; Xia, J. H. Chem Rev 2001, 101, 2921-2990.

5 Becer, C. R.; Paulus, R. M.; Hoeppener, S.; Hoogenboom, R.; Fustin, C.-A.; Gohy, J.-F.; Schubert, U. S. Macromolecules 2008, 41, 5210-5215.

6 Karas, M.; Hillenkamp, F. Anal Chem 1988, 60, 2299-2301.

7 Tanaka, K.; Waki, H.; Ido, Y.; Akita, S.; Yoshida, Y.; Yoshida, T. Rapid Commun Mass Spectrom 1988, 2, 151-153.

8 Fenn, J. B.; Mann, M.; Meng, C. K.; Wong, S. F.; Whitehouse, C. M. Science 1989, 246, 64-71.

9 Nielen, M. W. F. Mass Spectrom Rev 1999, 18, 309-344.

10 Hanton, S. D. Chem Rev 2001, 101, 572-569.

11 Montaudo, G.; Samperi, F.; Montaudo, M. S. Prog Polym Sci 2006, 31, 277-357.

12 Grunendling, T.; Weidner, S.; Falkenhagen, J.; BarnerKowollik, C. Polym Chem 2010, 1, 599-617.

13 McEwen, C. N.; Peacock, P. M. Anal Chem 2002, 74, 2743-2748.

14 Peacock, P. M.; McEwen, C. N. Anal Chem 2004, 76, 3417-3428.

15 Peacock, P. M.; McEwen, C. N. Anal Chem 2006, 78, 3957-3964.

16 Weidner, S. M.; Trimpin, S. Anal Chem 2008, 80, 4349-4361.

17 Crecelius, A. C.; Baumgaertel, A.; Schubert, U. S. J Mass Spectrom 2009, 44, 1277-1286.

18 Baumgaertel, A.; Weber, C.; Knop, K.; Crecelius, A.; Schubert, U. S. Rapid Commun Mass Spectrom 2009, 23, 756-762.

19 Baumgaertel, A.; Becer, C. R.; Gottschaldt, M.; Schubert, U. S. Macromol Rapid Commun 2008, 29, 1309-1315.

20 Knop, K.; Jahn, B. O.; Hager, M. D.; Crecelius, A.; Gottschaldt, M.; Schubert, U. S. Macromol Chem Phys 2010, 6, 677-684.

21 Wesdemiotis, C.; Pingitore, F.; Polce, M. J.; Russell, V. M.; Kim, Y.; Kausch, C. M. T.; Connors, H.; Medsker, R. E.; Thomas, R. R. Macromolecules 2006, 39, 8369-8378.

22 Weidner, S. M.; Falkenhagen, J.; Maltsev, S.; Sauerland, V.; Rinken, M. Rapid Commun Mass Spectrom 2007, 21, 2750-2758.

23 Weidner, S. M.; Falkenhagen, J.; Knop, K.; Thünemann, A. Rapid Commun Mass Spectrom 2009, 23, 2768-2774. 
24 Adamus, G. Rapid Commun Mass Spectrom 2007, 21, 2477-2490.

25 Terrier, P.; Buchmann, W.; Desmazieres, B.; Tortajada, J. Anal Chem 2006, 78, 1801-1806.

26 Simonsick, W. J.; Petkovska, V. I. Anal Bioanal Chem 2008, 392, 575-583.

27 Girod, M.; Phan, T. N. T.; Charles, L. J Am Soc Mass Spectrom 2008, 19, 1163-1175.

28 Girod, M.; Phan, T. N. T.; Charles, L. Rapid Commun Mass Spectrom 2008, 22, 3767-3775.

29 Girod, M.; Phan, T. N. T.; Charles, L. Rapid Commun Mass Spectrom 2009, 23, 1476-1482.

30 Giordanengo, R.; Viel, S.; Hidalgo, M; Allard-Breton, B.; Thévand, A.; Charles, L. Anal Chim Acta 2009, 654, 49-58.

31 Girod, M.; Mazarin, M.; Phan, T. N. T.; Gigmes, D.; Charles, L. J Polym Sci Part A: Polym Chem 2009, 47, 3380-3390.
32 Hoteling, A. J.; Kawaoka, K.; Goodberlet, M. C.; Yu, W.-M.; Owens, K. G. Rapid Commun Mass Spectrom 2003, 17, 1671-1676.

33 Selby, T. L.; Wesdemiotis, C.; Lattimer, R. P. J Am Soc Mass Spectrom 1994, 5, 1081-1092.

34 Lattimer, R. P.; Münster, H.; Budzikiewicz, H. Int J Mass Spectrom Ion Processes 1989, 90, 119-129.

35 Borman, C. D.; Jackson, A. T.; Bunn, A.; Cutter, A. L.; Irvine, D. J. Polymer 2000, 41, 6015-6020.

36 Gies, A. P.; Vergne, M. J.; Orndorff, R. L.; Hercules, D. M. Macromolecules 2007, 40, 7493-7504.

37 Polce, M. J.; Ocampo, M.; Quirk, R. P.; Wesdemiotis, C. Anal Chem 2008, 80, 347-354.

38 Polce, M. J.; Ocampo, M.; Quirk, R. P.; Leigh, A. M.; Wesdemiotis, C. Anal Chem 2008, 80, 355-362. 